\title{
The role of the microbiology laboratory in the investigation of child sexual abuse
}

\author{
C. DYSON and I. K. HOSEIN \\ Department of Medical Microbiology and Public Health Laboratory, University Hospital of Wales, Heath Park, \\ Cardiff CF 4 XW
}

\begin{abstract}
Detection of a sexually transmitted pathogen in a child is suggestive of sexual abuse. Consequently, there are very strong clinical, social and legal reasons for diagnosing sexually transmitted disease in children correctly. Carefully considered protocols should be established for all stages of the microbiological investigation. All procedures, from specimen collection to report generation, should be undertaken to the highest possible standard with appropriate documentation. For the more commonly identified sexually transmitted pathogens in the paediatric population, the gold standard of diagnosis in the microbiology laboratory remains culture in vitro because this method offers maximum specificity. Whenever possible, culture must be followed by appropriate confirmatory tests. This highly exacting approach has significant resource and organisational implications and some tests may have to be centralised. The effective provision of an appropriate service for these cases is dependent on the local laboratory collaborating closely with hospital paediatricians and other microbiology laboratories.
\end{abstract}

\section{Introduction}

It is now accepted that the possibility of child sexual abuse (CSA) should be considered whenever a sexually transmitted pathogen is detected in a child. The diagnosis of CSA has far reaching medical, psychosocial and legal implications and, consequently, the clinical management of suspected cases requires great care and attention to detail. The detection of a sexually transmitted pathogen may lead to familial or official intervention, or both, that could improve the child's social circumstances. Furthermore, the presence of sexually transmitted pathogens may be used as supportive evidence of CSA in a Court of Law. On the other hand, misleading results may have very damaging consequences. False-positive results may trigger management decisions that cause unnecessary psychosocial stress to child and family members and, additionally, may render the hospital liable to legal action. Conversely, false-negative results may inadvertently allow a continuation of abuse. These considerations dictate that tests for the microbiological investigation of suspected CSA are performed to the highest possible standard, that procedures are properly documented, and that laboratory methods have maximum sensitivity and specificity.

Received 9 Jan: 1996; revised version received 16 April 1996 accepted 22 April 1996.

Corresponding author: Dr C. Dyson.

\section{Epidemiological background}

Most epidemiological studies on sexually transmitted disease and CSA have originated from the USA and these have identified a clear relationship between sexual abuse and infection with the sexually transmitted pathogens Neisseria gonorrhoeae [1-5], Treponema pallidum [1,5], Chlamydia trachomatis [5], Trichomonas vaginalis [1] and human papilloma virus (HPV) [5]. Published case reports on infection with herpes simplex virus (HSV) [6, 7], HPV [8] and human immunodeficiency virus (HIV) [9] in sexually abused children suggest the same association. Although epidemiological data are currently unavailable, there is concern that the prevalence of HIV infection in sexually abused children in the USA is increasing [10]. The role of sexual spread in the aetiology of infection by Gardnerella vaginalis and Mycoplasma hominis is less clear, but some studies have demonstrated a higher rate of detection of these organisms in groups of abused children compared with control groups [11-13]. UK data on sexually transmitted disease (STD) in CSA are scarce, but it is reasonable to assume that a similar association exists. This relationship implies that the diagnosis of a STD in a child is suggestive of CSA, and it has been shown that historical evidence of CSA can be obtained in the majority of cases of infection with $N$. gonorrhoeae [5], C. trachomatis [5], HSV [7] and HPV [5]. 
Non-sexual spread of a sexually transmitted pathogen may give rise occasionally to infection in children. Antenatal and perinatal transmission are recognised modes of spread in many infections caused by sexually transmitted pathogens. The debate over the role of fomites in the transmission of sexually transmitted disease has been long running. $N$. gonorrhoeae can survive in secretions for up to $74 \mathrm{~h}$, depending on the type of surface [14], suggesting that indirect spread may, at least theoretically, give rise to infection. However, there is no convincing evidence of spread by fomites and sexual transmission is considered to be the predominant mode of transmission. Similarly, T. vaginalis can survive on damp fabrics for several hours [15] and, although non-sexual spread is theoretically possible, sexual contact is considered to be the most likely cause of infection with this organism. Spread by fomites has also been implicated in molluscum contagiosum [16].

\section{Laboratory investigation}

\section{Specimen collection}

A recent report [17] recommended investigation for $N$. gonorrhoeae, $C$. trachomatis, $T$. vaginalis and $T$. pallidum in all cases, combined with additional investigations for other pathogens when clinical findings suggest their presence. The same report recommended specimen collection from multiple body sites in all cases of suspected CSA. Vaginal (or penile), rectal and pharyngeal specimens were recommended for $N$. gonorrhoeae investigation, vaginal (or penile) and rectal specimens for $C$. trachomatis, and a vaginal specimen only for $T$. vaginalis. Additionally, a serum specimen was advised for $T$. pallidum antibody tests and storage for possible future hepatitis B virus (HBV) or HIV testing, or both. The importance of properly timed repeat investigations, given that recently acquired infections may escape detection at the initial screen, has been emphasised [18]. Extensive investigation involving careful specimen collection is traumatic for the child and has a low yield, but the justification for a painstaking approach is that infection in CSA is often asymptomatic, an accurate history is difficult to obtain and a positive result has highly significant implications.

A local policy for the routine microbiological investigation of CSA should be drawn up by a group comprising paediatricians and medical microbiologists, and made available to all hospital personnel involved in the investigation of CSA cases. It is the responsibility of this group to define the extent of investigation in an asymptomatic case. It seems reasonable, taking into account the balance of probability and anticipated yield, that investigation could be limited in asymptomatic children to tests for $N$. gonorrhoeae, $C$. trachomatis and $T$. vaginalis (Table 1). However, the policy should indicate possible additional microbiological investigations depending on individual social circumstances and clinical features (Table 2). Availability at all times, at the point of use, of appropriate swabs and transport media is central to the successful implementation of such a policy.

Legal considerations dictate that there should be

Table 1. Core investigations performed in all cases of suspected CSA

\begin{tabular}{|c|c|c|}
\hline Specimen type & Pathogen & Method(s) of investigation \\
\hline Vaginal swab in general transport medium & N. gonorrhoeae & $\begin{array}{l}\text { Microscopy of gram-stained smear } \\
\text { Culture on gonococcal medium for } 2 \text { days } \\
\text { Gram's stain and oxidase test on suspicious } \\
\text { colonies } \\
\text { Confirmation of suspicious colony by at least two } \\
\text { unrelated tests } \\
\beta \text {-Lactamase test on colony } \\
\text { Sensitivity tests }\end{array}$ \\
\hline & T. vaginalis & Microscopy of wet mount \\
\hline Vaginal swab in chlamydia transport medium & C. trachomatis & Cell culture for 3 days \\
\hline Vaginal swab in trichomonas culture medium & T. vaginalis & $\begin{array}{l}\text { Culture in trichomonas culture medium for } 7 \\
\text { days }\end{array}$ \\
\hline Smear on slide & C. trachomatis & Direct immunofluorescence \\
\hline
\end{tabular}

Table 2. Additional investigations indicated on the basis of clinical findings and/or social circumstances

\begin{tabular}{lll}
\hline Specimen type & Pathogen & Method(s) of investigation \\
\hline Venous blood & Hepatitis B virus & Specific tests for antibody \\
& Human immunodeficiency virus & Protocols as for adult specimens \\
Specimens from lesions & $T$. pallidum & Cell culture followed by confirmation \\
& Herpes simplex virus & Usually a clinical diagnosis \\
& Molluscum contagiosum & Light microscopy or electron microscopy may be \\
helpful & as in Table 1 \\
Rharyngeal swab & & as in Table 1 \\
& N. gonorrhoeae & Cell culture for 3 days \\
\hline
\end{tabular}


evidence of continuity of transfer of specimens from the time of collection to receipt by the laboratory. This implies that clinical or laboratory staff should deliver specimens personally to the laboratory, and that the identity of individuals involved in this stage of the investigation should be recorded.

\section{Specimen processing}

Quality assurance is a major consideration in the dayto-day management of a pathology laboratory, to the extent that staff should emphasise the need for great care in the processing of all specimens irrespective of the clinical context. CSA specimens merit special attention for the reasons mentioned above, and laboratory protocols should give clear instructions on the handling of specimens, performance of tests and appropriate action to be taken when a positive result is obtained.

Work done on each specimen should be documented at all stages of the investigation. This documentation should include records of the date and time that the specimen is received by the laboratory and of the tests performed. Given that the results of laboratory investigations may be used as legal evidence, the identity of key individuals involved in laboratory investigation of the specimens should be documented. All laboratory tests should be performed by experienced personnel. Maintaining confidentiality at all stages of the investigation is essential and, in line with this, reports should be despatched in confidential envelopes addressed to the clinical consultant or another authorised individual.

All request forms, laboratory records, serum specimens and isolates should be stored for an agreed period of time. Sending isolates to reference laboratories for independent confirmation should be considered [18].

\section{Choice of methods}

Medical, psychosocial and legal considerations dictate that laboratory methodology should be as reliable and accurate as possible. $N$. gonorrhoeae, $C$. trachomatis, T. vaginalis and HSV are routinely cultivable sexually transmitted pathogens and the gold standard methodology for their detection is culture. This methodology offers the opportunity to achieve maximum test specificity. In addition, the use of culture (followed, when appropriate, by confirmation) minimises the risk of false-positive results that may arise when other methods are used. Furthermore, contamination of the vagina with oral and faecal flora, which occurs readily in younger age groups because of imperfect hygiene, re-inforces the importance of using highly specific tests in this age group. Culture methods also offer high sensitivity; in suspected CSA cases there are potential difficulties in obtaining specimens containing sufficient numbers of organisms because the traumatised child may not comply readily during specimen collection. Antigen detection tests for $N$. gonorrhoeae, $C$. trachomatis and HSV in genital tract specimens have not been licensed for use in children although they are used widely in adults. Nor have these tests been licensed for use on rectal or pharyngeal swabs in any patient, irrespective of age. Gene probe assays for $N$. gonorrhoeae and $C$. trachomatis [19] are becoming used more widely in adults but, again, are not licensed for use in children. A further important advantage of culture is that it provides an opportunity for typing isolates obtained from the child and alleged perpetrator which may provide valuable clinical and legal evidence.

$N$. gonorrhoeae. In adult cases of gonococcal infection, light microscopy of a smear with Gram's stain yields valuable presumptive information. The sensitivity with Gram's stain of urethral discharge in male urethritis is very high, but is much lower in females when endocervical secretions are examined [20]. The presence of intracellular gram-negative diplococci in the urethral discharge of sexually active adult males has a high predictive value and treatment may be instituted on the basis of this result. However, the predictive value of this finding is reduced in low risk patients, particularly children in whom contamination of the genital area by oropharyngeal organisms (including Neisseria spp.) may occur as a result of auto-inoculation. Therefore a smear examined with Gram's stain has little to offer in the investigation of suspected CSA. Culture on a selective medium, followed by thorough confirmatory testing, is central to the diagnosis of gonococcal infection in these cases [21].

Despite the inclusion of selective agents, $N$. meningitidis [22], N. lactamica [22], N. polysaccharea [23], Kingella denitrificans [24] and some strains of $N$. cinerea [24] are able to grow on commonly used gonococcal culture media. Strains of these species may resemble $N$. gonorrhoeae microscopically and macroscopically and, like $N$. gonorrhoeae, will give rise to a positive oxidase reaction. Further confirmatory testing is, therefore, essential for the routine identification of $N$. gonorrhoeae, and three different methodologies-carbohydrate utilisation, enzyme detection and immunological tests - are available routinely. None of these tests used in isolation is adequate for confirmation of $N$. gonorrhoeae, and these tests are, therefore, usually performed in combination.

In the carbohydrate utilisation tests [25], Neisseria spp. produce a characteristic pattern of acid formation from bacterial action on a range of sugars, including glucose, maltose, lactose and sucrose. $N$. gonorrhoeae degrades glucose only, and therefore cannot be distinguished from $K$. denitrificans which produces identical reactions with the commonly used carbo- 
hydrates [26]. Some strains of $N$. gonorrhoeae are weak producers of acid from glucose and may not produce a detectable positive result in the glucose test [27], making them indistinguishable from unreactive species such as $N$. cinerea, N. flava and Branhamella catarrhalis (sic). Conversely, some strains of $N$. meningitidis are maltose-negative and may be misidentified as $N$. gonorrhoeae [28].

Chromogenic enzyme substrate tests enable differentiation of strains by detection of species-specific enzymes [20]. N. gonorrhoeae possesses the hydroxyprolylaminopeptidase enzyme [29], as do some nonpathogenic Neisseria spp. including $N$. cinerea [24] and $K$. denitrificans [24].

The two immunological methods that are used most commonly for cultural confirmation are fluorescent antibody [30] and co-agglutination [31] tests. These tests incorporate a monoclonal antibody (MAb) specific to several gonococcal $1 \mathrm{~A}$ and $1 \mathrm{~B}$ proteins. Although the introduction of MAbs in place of polyclonal antibodies has improved the specificity and sensitivity of both methods greatly [20], problems have been encountered when these tests have been used. Cross-reactions have been observed with other Neisseria spp. in the coagglutination tests; thus in one commercial test (Phadebact Monoclonal GC OMNI Test) [32] cross-reactions were observed when $N$. lactamica, $N$. cinerea and $B$. catarrhalis (sic) were tested, while another test (GonoGen) [32] gave a falsepositive result with $N$. meningitidis. Despite proven high sensitivity, there is concern that these MAb-based methods may fail to detect strains with novel epitopes [32]. Moreover, the failure of a penicillinase-producing strain of $N$. gonorrhoeae to fluoresce has been reported [33].

In an investigation of 40 paediatric isolates that were identified initially as $N$. gonorrhoeae by clinical microbiology laboratories in the USA, 14 were found to belong to species other than $N$. gonorrhoeae when tested at a reference laboratory [21]. Of these isolates, four were identified as $N$. cinerea, three as $M$. catarrhalis, three as $N$. lactamica, one as $K$. denitrificans and one was unidentifiable. The results of this investigation underline the importance of thorough and accurate confirmatory testing and the authors recommended the use of at least two confirmatory methods.

C. trachomatis. Immunological methods have become increasingly popular in the diagnosis of C. trachomatis infection in adults, and ELISA has an established role as a screening test for chlamydia in adult STD clinic patients. The sensitivity of this method is low $[34,35]$, but may be enhanced by multiple swabbing [35]. However, this approach is traumatic for children and the achievable level of sensitivity remains unacceptable in the investigation of suspected CSA. The specificity of the test is higher than its sensitivity [34, 35]. However, the positive predictive value is reduced in a low prevalence population; this renders the test unacceptable in low risk cases [36]. False-positive results may result from the presence in the anogenital flora of organisms (Escherichia coli, Acinetobacter calcoaceticus, N. gonorrhoeae, group B streptococci and $G$. vaginalis) that cross-react with chlamydiae [34]. When rectal specimens are tested, non-specific binding of unknown faecal constituents to mouse immunoglobulin is responsible for some false-positive results [37]. Moreover, faecal contamination of the genital area is more likely to occur in children.

The sensitivity and specificity of the direct immunofluorescence (DIF) test are higher than those of the ELISA test [38]. However, in the DIF test, nonspecific binding of $\mathrm{Fc}$ receptor-bearing protein to labelled antibody may render some staphylococcal species fluorescent [39]. Furthermore, the presence of fluorescing artefactual bodies [40], which are seen most commonly in rectal specimens, may also give rise to false-positive results, particularly in inexperienced hands. The sensitivity of the DIF test is low [41-43]; in a study of abused children [41] and a study of 'at risk' children [42], the yield from the DIF test was considerably lower than that from cell culture.

Cell culture is more specific than antigen detection tests and is the method of choice in CSA cases, given the medico-legal dimension to these cases [36]. Antigen detection tests should not be used alone in this context; low test specificity means that positive results would not be acceptable in a Court of Law [36]. However, use of the DIF test, in conjunction with cell culture, is justified because the adequacy of the specimen can be noted readily during microscopic visualisation of the smear. This feature of the test is of particular value in paediatric investigation because of potential difficulties in obtaining adequate samples. Furthermore, DIF-positive yet culture-negative results are a recognised finding [43]; these should then be a trigger of repeat culture for $C$. trachomatis.

$T$. vaginalis. In the detection of $T$. vaginalis, direct microscopy of a wet mount film prepared from a vaginal swab has modest sensitivity, but very high specificity [44]. Culture in a liquiid medium is the most sensitive method [45] for the detection of $T$. vaginalis, but it is an imperfect gold standard with sub-optimal sensitivity [45]. Sensitivity seems to be dependent, at least partly, on inoculum size, which may be small in paediatric specimens [45]. Wet mount microscopy is less sensitive [44], but has a potentially valuable role because a wet mount-positive result in the face of negative culture is an occasional finding [44]. There are differences in sensitivity between different media; Diamond's 'medium and modified Diamond's medium perform better than other media [46]. 
Herpes simplex virus. Immunologically based methods-especially DIF [47, 48] and ELISA [49]-have, as in the case of $C$. trachomatis, an established role in the diagnosis of genital herpes in adults but, again, these methods are not suitable in paediatric cases. The sensitivities and specificities of both methods are high [47-49], but not high enough to justify their use in CSA investigation. Cell culture for HSV remains the gold standard method for the investigation of suspected CSA cases [50]. Confirmatory tests are essential in identification of HSV since a cytopathic effect resembling that of HSV may occur when other viruses or substances such as urine or semen are present in the specimen [51].

Other pathogens. Serological methods have an established role in the investigation of $T$. pallidum, HBV and HIV infection in adults. These methods are generally very reliable for these infections and are suitable for use in the CSA context with appropriate indications and counselling. In keeping with the need to exercise great care in CSA cases, further serum specimens should be tested for confirmation if a positive result is obtained. Molluscum contagiosum is usually diagnosed on clinical examination but, where there is doubt, smears of lesion material may be examined for inclusion bodies by light microscopy, or for viral particles by electron microscopy. Genital warts are diagnosed clinically and the routine laboratory has, at present, nothing to offer in terms of microbiological confirmation or typing. However, when local symptoms and signs are present, investigations for other bacterial pathogens should be performed according to normal laboratory protocols. Diagnosis of bacterial vaginosis is clinically important because it is a treatable condition. However, this condition has little forensic significance, given its weak association with CSA, and culture for $G$. vaginalis cannot be considered mandatory. A laboratory that uses microscopy only to diagnose bacterial vaginosis has no reason to change its protocol for CSA cases.

\section{Organisational considerations}

The adoption of cultural techniques for CSA investigation will have organisational implications for many laboratories. Culture for $N$. gonorrhoeae is part of the repertoire of all diagnostic laboratories, and properly controlled confirmatory tests on isolates are an established part of the investigation. Some laboratories may have to arrange additional confirmatory test(s) for this organism, and this would mean sending the isolate to another laboratory. Culture of C. trachomatis and HSV involves the use of cell culture methods, but these are unavailable in most district general hospital laboratories. This implies that CSA specimens would have to be referred in many cases to laboratories that are able to provide a cell culture service. The laboratories which perform only direct microscopy for $T$. vaginalis may consider adopting a culture method or referring the specimen to another laboratory. Provision of a centralised culture service for these pathogens is acceptable if appropriate transport media are available at the point of use and if there is no undue delay in transport. Infections by $T$. pallidum, HBV and HIV are diagnosed almost always by serology, and this methodology is within the capability of a routine laboratory, but also lends itself to centralisation.

\section{Conclusions}

The microbiological investigation of suspected CSA cases demands laboratory practices that are carefully considered and optimal. There should be close liaison with paediatricians and a high level of technical expertise in the laboratory. The performance of reliable and accurate tests should be accompanied by adherence to correct procedures in all cases. Correctly made laboratory diagnoses may alter management to the benefit of the child, yet the laboratory may still have to defend its procedures, methods and results in a Court of Law. Erroneous results may lead to inappropriate management decisions, with potentially damaging consequences for individuals concerned and for the hospital, which may face subsequent legal action. It is imperative that the risk of misidentification in the laboratory is minimised as far as possible and that procedures undertaken in suspected CSA cases are clinically optimal and legally unassailable.

The use of routine cultural methods in suspected cases of CSA is essential because these methods come closest to fulfilling exacting clinical and legal requirements. Thus standards for methods of investigation for $N$. gonorrhoeae, $C$. trachomatis, $T$. vaginalis and $\mathrm{HSV}$ in suspected CSA cases differ from what is considered acceptable in adult cases.

There are significant organisational and resource implications in introducing and providing an appropriate service for CSA cases, but these are not insurmountable. However, it is clear that effective microbiological investigation by the laboratory of these difficult but extremely important cases depends on close collaboration with the paediatric department and, in many cases, with other microbiology laboratories.

\section{References}

1. White ST, Loda FA, Ingram DL, Pearson A. Sexually transmitted diseases in sexually abused children. Pediatrics 1983; 72: 16-21.

2. De Jong A. Sexually transmitted diseases in sexually abused children. Sex Transm Dis 1986; 13: 123-126.

3. Rimsza ME, Niggemann EH. Medical evaluation of sexually abused children: a review of 311 cases. Pediatrics 1982; 69: $8-14$.

4. Tilelli JA, Turek D, Jaffe AC. Sexual abuse of children. Clinical findings and implications for management. $N$ Engl J Med 1980; 302: 319-323. 
5. Ingram DL, Everett VD, Lyna PR, White ST, Rockwell LA. Epidemiology of adult sexually transmitted disease agents in children being evaluated for sexual abuse. Pediatr Infect Dis $J$ 1992; 11: 945-950.

6. Gardner M, Jones JG. Genital herpes acquired by sexual abuse of children. $J$ Pediatr 1984; 104: 243-244.

7. Kaplan KM, Fleischer GR, Paradise JE, Friedman HN. Social relevance of genital herpes simplex in children. Am J Dis Child 1984; 138: 872-874.

8. Seidel J, Zonana J, Totten E. Condylomata acuminata as a sign of sexual abuse in children. $J$ Pediatr 1979; 95: 553-554.

9. Gutman LT, St Claire KK, Weedy $\mathrm{C}$ et al. Human immunodeficiency virus transmission by child sexual abuse Am J Dis Child 1991; 145: 137-141.

10. Gellert GA, Durfee MJ. HIV infection and child abuse. $N$ Engl $J$ Med 1989; 321: 685.

11. Bartley DL, Morgan L, Rimsza ME. Gardnerella vaginalis in prepubertal girls. Am J Dis Child 1987; 141: 1014-1017.

12. Steele AM, de San Lazaro C. Transhymenal cultures for sexually transmissible organisms. Arch Dis Child 1994; 71: $423-427$.

13. Hammerschlag MR, Doraiswamy B, Cox $\mathrm{P}$, Cummings $\mathrm{M}$, McCormack WM. Colonization of sexually abused children with genital mycoplasmas. Sex Transm Dis 1987; 14: 23-25.

14. Srivastava AC. Survival of gonocoocci in urethral secretions with reference to the nonsexual transmission of gonococcal infection. $J$ Med Microbiol 1980; 13: 593-596.

15. Catterall RD, Nicol CS. Is trichomonal infestation a venereal disease? BMJ 1960; 1: 1177-1179.

16. Brown ST, Nalley JF, Kraus SJ. Molluscum contagiosum. Sex Transm Dis 1981; 8: 227-234.

17. Royal College of Physicians Working Party. Physical signs of sexual abuse in children. London, RCP. 1991.

18. Schwarcz SK, Whittington WL. Sexual assault and sexually transmitted diseases: detection and management in adults and children. Rev Infect Dis 1990; 12 Suppl 6: S682-S690.

19. Hosein IK Kaunitz AM, Craft SJ. Detection of cervical Chlamydia trachomatis and Neisseria gonorrhoeae with deoxyribonucleic acid probe assays in obstetric patients. $\mathrm{Am}$ $J$ Obstet Gynecol 1992; 167: 588-591.

20. Easmon CSF, Ison CA. Neisseria gonorrhoeae: a versatile pathogen. J Clin Pathol 1987; 40: 1088-1097.

21. Whittington WL, Rice RJ, Biddle JW, Knapp JS. Incorrect identification of Neisseria gonorrhoeae from infants and children. Pediatr Infect Dis 1988; 7: 3-10.

22. Morello JA, Janda WM, Doern GV. Neisseria and Branhamella. In: Balows A, Hausler WJ, Herrmann KL, Isenberg $\mathrm{HD}$ Shadomy HJ (eds) Manual of clinical microbiology. Washington, DC, American Society for Microbiology, 1991; 258-276.

23. Riou J-Y, Guibourdenche M. Neisseria polysaccharea $\mathrm{sp}$ nov Int J Syst Bacteriol 1987; 37: 163-165.

24. Knapp JS. Historical perspectives and identification of Neisseria and related species. Clin Microbiol Rev 1988; 1: 415-431.

25. Kellogg DS, Turner EM. Rapid fermentation confirmation of Neisseria gonorrhoeae. Appl Microbiol 1973; 25: 550-552.

26. Hollis DG, Weaver RE, Riley PS. Emended description of Kingella denitrificans (Snell and Lapage 1976): correction of the maltose reaction. $J$ Clin Microbiol 1983; 18: 1174-1176.

27. Morello JA, Lerner SA, Bohnhoff M. Characteristics of atypical Neisseria gonorrhoeae from disseminated and localised infections. Infect Immun 1976; 13: 1510-1516.

28. Granato PA, Howard R, Wilkinson B, Laser J. Meningitis caused by maltose-negative variant of Neisseria meningitidis $J$ Clin Microbiol 1980; 11: 270-273.

29. D'Amato RF, Eriquez LA, Tomfohrde KM, Singerman E. Rapid identification of Neisseria gonorrhoeae and Neisseria meningitidis by using enzymatic profiles. $J$ Clin Microbiol 1978; 7: 77-81.

30. Laughon BE, Ehret JM, Tanino TT et al. Fluorescent monoclonal antibody for confirmation of Neisseria gonorrhoeae cultures. J Clin Microbiol 1987; 25: 2388-2390.

31. Carlson BL, Calnan MB, Goodman RE, George H. Phadebact Monoclonal GC OMNI Test for confirmation of Neisseria gonorrhoeae. J Clin Microbiol 1987; 25: 1982-1984.
32. Dillon JR, Carballo M, Pauze M. Evaluation of eight methods for identification of pathogenic Neisseria species: Neisseria Kwik, RIM-N, Gonobio-Test, Minitek, Gonochek II, GonoGen, Phadebact Monoclonal GC OMNI Test, and Syva Microtrak Test. J Clin Microbiol 1988; 26: 493-497.

33. Walton DT. Fluorescent-antibody-negative penicillinase-producing Neisseria gonorrhoeae. J Clin Microbiol 1989; 27: 1885 1886

34. Taylor-Robinson D, Thomas BJ, Osborn MF. Evaluation of enzyme immunoassay (Chlamydiazyme) for detecting Chlamydia trachomatis in genital tract specimens. J Clin Pathol 1987, 40: 194-199.

35. Thomas BJ, Osborn MF, Gilchrist C, Taylor-Robinson D. Improved sensitivity of an enzyme immunoassay IDEIA for detecting Chlamydia trachomatis. J Clin Pathol 1989; 42: 759 762 .

36. Hammerschlag MR, Rettig PJ, Shields ME. False positive results with the use of chlamydia antigen detection tests in the evaluation of suspected sex abuse in children. Pediatr Infect Dis $J$ 1988; 7: 11-14

37. Riordan T, Ellis DA, Matthews PI, Ratcliffe SF. False positive results with an ELISA for detection of chlamydia antigen. $J$ Clin Pathol 1986; 39: 1276-1277.

38. Tam MR, Stamm WE, Handsfield HH et al. Cultureindependent diagnosis of Chlamydia trachomatis using monoclonal antibodies. $N$ Engl J Med 1984; 310: 1146-1150.

39. Krech T, Gerhard-Fsadni D, Hofmann N, Miller SM. Interference of Staphylococcus aureus in the detection of Chlamydia trachomatis by monoclonal antibodies. Lancet 1985; 1: 1161-1162.

40. Rompalo AM, Suchland RJ, Price CB, Stamm WE. Rapid diagnosis of Chlamydia trachomatis rectal infection by direct immunofluorescence staining. J Infect Dis 1987; 155: 1075 1076.

41. Fuster CD, Neinstein LS. Vaginal Chlamydia trachomatis prevalence in sexually abused prepubertal girls. Pediatrics 1987; 79: 235-238.

42. Hauger SB, Brown J, Agre F, Sahrail F, Ortiz R, Ellner P. Failure of direct fluorescent antibody staining to detect Chlamydia trachomatis from genital tract sites of prepubertal children at risk for sexual abuse. Pediatr Infect Dis $J$ 1988; 7 : 660-662.

43. Thejls $\mathrm{H}$, Gnarpe J, Gnarpe $\mathrm{H}$ et al. Expanded gold standard in the diagnosis of Chlamydia trachomatis in a low prevalence population: diagnostic efficacy of tissue culture, direct immunofluorescence, enzyme immunoassay, PCR and serology. Genitourin Med 1994; 70: 300-303.

44. Krieger JN, Tam MR, Stevens CE et al. Diagnosis of trichomoniasis. Comparison of conventional wet-mount examination with cytologic studies, cultures, and monoclonal antibody staining of direct specimens. JAMA 1988; 259: $1223-1227$

45. Lossick JG. The diagnosis of vaginal trichomoniasis. JAMA 1988; 259: 1230

46. Schmid GP, Matheny LC, Zaidi AA, Kraus SJ. Evaluation of six media for the growth of Trichomonas vaginalis from vaginal secretions. $J$ Clin Microbiol 1989; 27: 1230-1233.

47. Lafferty WE, Krofft S, Remington $\mathrm{M}$ et al. Diagnosis of herpes simplex virus by direct immunofluorescence and viral isolation from samples of external genital lesions in a high-prevalence population. $J$ Clin Microbiol 1987; 25: 323-326.

48. Pouletty P, Chomel JJ, Thouvenot D, Catalan F, Rabillon V, Kadouche J. Detection of herpes simplex virus in direct specimens by immunofluorescence assay using a monoclonal antibody. J Clin Microbiol 1987; 25: 958-959.

49. Dascal A, Chan-Thim J, Morahan M, Portnoy J, Mendelson J. Diagnosis of herpes simplex virus infection in a clinical setting by a direct antigen detection enzyme immunoassay kit. $J$ Clin Microbiol 1989; 27: 700-704.

50. Ashley RL. Laboratory techniques in the diagnosis of herpes simplex infection. Genitourin Med 1993; 69: 174-183.

51. Ashley RL. Genital herpes infections. Clinics in laboratory medicine. In: Judson FN (ed) Sexually transmitted diseases. Philadelphia, WB Saunders. 1989: 405-420. 